\title{
The ABCs of successful vaccinations: A role for psychiatry
}

\author{
Oliver Freudenreich, MD, FACLP, Manjola U. Van Alphen, MD, PhD, MBA, and Carol Lim, MD, MPH
}

Dr. Freudenreich is Co-Director, Schizophrenia Clinical and Research Program, Massachusetts General Hospital, Boston, Massachusetts. Dr. Van Alphen is CMO of North Suffolk Mental Health Association, Chelsea, Massachusetts. Dr. Lim is a Fellow in Public and Community Psychiatry, Massachusetts General Hospital, Boston, Massachusetts.

\section{Disclosures}

The authors report no financial relationships with any companies whose products are mentioned in this article, or with manufacturers of competing products.

doi: 10.12788/cp.0108

\section{f}

Discuss this article at www.facebook.com/ MDedgePsychiatry (
W hile the implementation of mass vaccinations is a public health task, individual clinicians are critical for the success of any vaccination campaign. Psychiatrists may be well positioned to help increase vaccine uptake among psychiatric patients. They see their patients more frequently than primary care physicians do, which allows for patient engagement over time regarding vaccinations. Also, as physicians, psychiatrists are a trusted source of medical information, and they are well-versed in using the tools of nudging and motivational interviewing to manage ambivalence about receiving a vaccine (vaccine hesitancy). ${ }^{1}$

The "ABCs of successful vaccinations" (Figure) provide a framework that psychi- atrists can use when speaking with their patients about vaccinations. The ABCs assess psychological factors that hinder acceptance of vaccination (A = Attitudes toward vaccination), practical challenges in vaccine access for patients who are willing to get vaccinated ( $\mathrm{B}=$ Barriers to vaccination), and the actual outcome of "shot in the $\operatorname{arm}^{\prime \prime}(\mathrm{C}=$ Completed vaccination series). The Figure provides examples of each area of focus.

\section{How to talk to patients about vaccines}

"Attitudes toward vaccination" is an area in which psychiatrists can potentially move patients from hesitancy to vaccine confidence and acceptance. First, express

\section{Figure}

\section{The $A B C s$ of successful vaccinations}

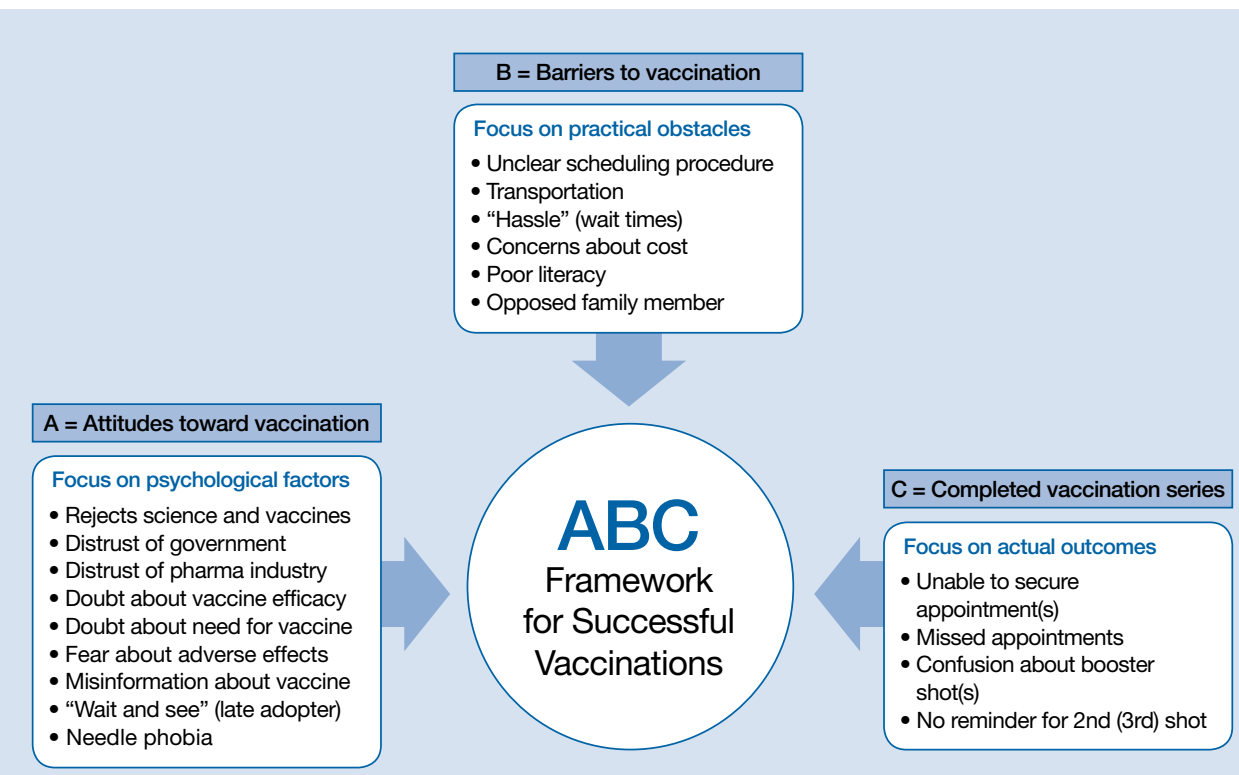


confidence in the vaccine (ie, make a clear statement: "You are an excellent candidate for this vaccine."). Then, begin a discussion using presumptive language: "You must be ready to receive the vaccine." In individuals who hesitate, elicit their concern: "What would make vaccination more acceptable?" In those who agree in principle about the benefits of vaccinations, ask about any impediments: "What would get in the way of getting vaccinated?" While some patients may require more information about the vaccine, others may need more time or mostly concrete help, such as assistance with scheduling a vaccine appointment. Do not to forget to follow up to see if a planned and complete vaccination series has taken place. The CDC offers an excellent online toolkit to help clinicians discuss vaccinations with their patients. ${ }^{2}$

Psychiatric patients, particularly those from disadvantaged and marginalized populations, have much to gain if psychiatrists are involved in preventive health care, including the coronavirus vaccination drive or the annual flu vaccination campaign.

\footnotetext{
References

1. McClure CC, Cataldi JR, O'Leary ST. Vaccine hesitancy: where we are and where we are going. Clin Ther. 2017; 39(8):1550-1562.

2. Centers for Disease Control and Prevention. COVID-19 vaccination toolkits. Accessed February 8, 2021. https: / / www.cdc.gov / coronavirus / 2019-ncov / vaccines/toolkits.html
}

\section{MARKETPLACE}

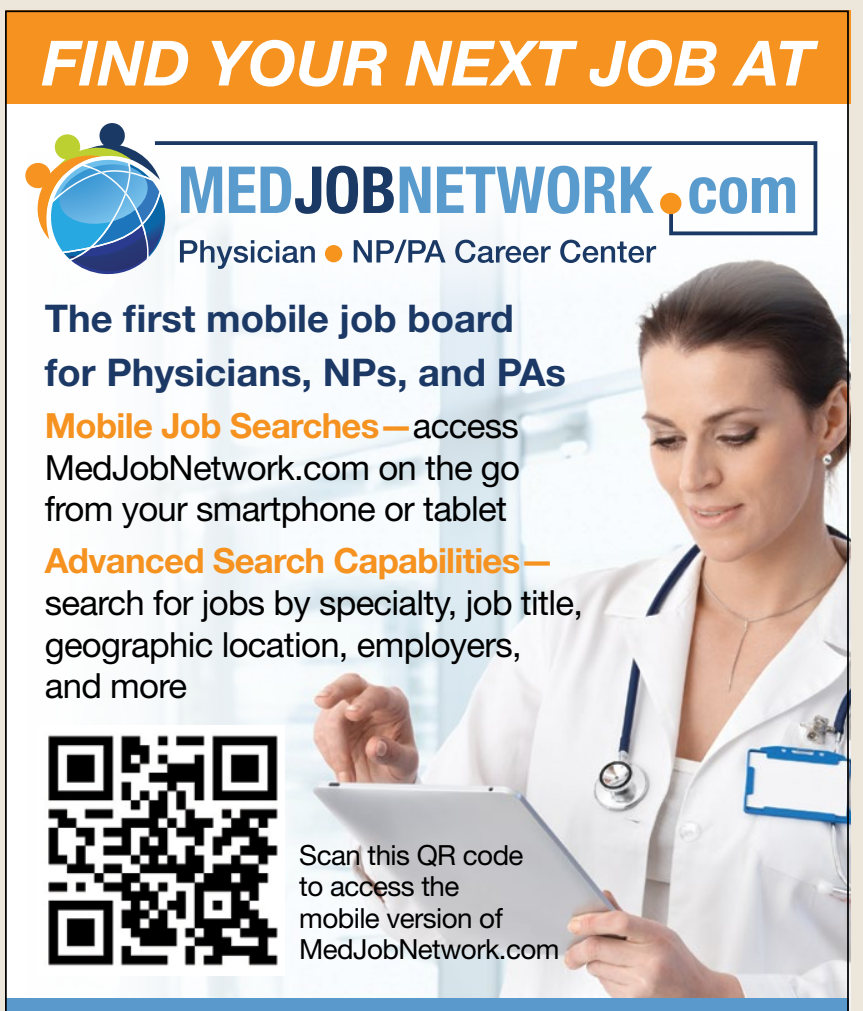

To advertise online contact: Tim LaPella

>484-921-5001 > tlapella@mdedge.com
MEDJOBNETWORK, com

Physician • NP/PA Career Center

\section{AD INDEX}

Intra-Cellular Therapies

Caplyta

PP 20-23

Janssen

Invega.

PP 1-9

Spravato C3-C4, PP 50-56

Menninger Clinic P 13

Supernus Pharmaceuticals, Inc P 11

United States Army. P 17

Washington Permanente Medical Group 